\title{
Negotiating Relation Work with Telehealth Home Care Companionship Technologies that Support Aging in Place
}

\author{
AMANDA LAZAR, University of Maryland, College Park, USA \\ HILAIRE J. THOMPSON, University of Washington, USA \\ SHIH-YIN LIN, University of Washington, USA \\ GEORGE DEMIRIS, University of Pennsylvania, USA
}

\begin{abstract}
In response to a perceived caregiver shortage and need to support aging in place, telehealth home care systems are being developed to provide remote care and monitoring to older people. Though research has examined the experiences of teleoperators delivering care through these systems, we know less about the experiences of older adults receiving this care. We report findings from a three-month study of a tablet-based telehealth home care system that provides support for aging in place. We find that there is a mismatch between the designer's conception of how care should be delivered and the ways that participants were interested in using the system. This mismatch exists in four areas: participants' interest in getting to know the human teleoperators and rejecting the virtual avatar, interrogating the interface to figure out the inner workings of the platform, pushing for a more symmetrical relationship, and negotiating the relation work that they were willing to perform in the "sacred space" of their homes. We draw on the concept of heteromation to understand the political dimensions of telehealth aging in place technologies. We also provide implications and future directions for technologies requiring relation work as well as the design of avatar-based remote companionship.
\end{abstract}

CCS Concepts: • Human-centered computing $\rightarrow$ Human-computer interaction (HCI); Collaborative and social computing;

\section{KEYWORDS}

Telecare, Older adults, Aging in place, Companion technologies

\section{ACM Reference format:}

Amanda Lazar, Hilare J. Thompson, Shih-Yin Lin, George Demiris. 2018. Negotiating Relation Work with Telehealth Home Care Companionship Technologies that Support Aging in Place. ACM Hum.-Comput. Interact. 2, CSCW, Article 103 (November 2018), 19 pages. DOI: https://doi.org/10.1145/3274372

\section{INTRODUCTION}

A much-discussed demographic shift is occurring, with a growing older population worldwide. This shift is attributed to dropping fertility rates, increased life expectancy, and demographic trends such as Baby Boomers. In response to this shift, there have been worldwide calls to cope with an aging population. Almost a decade ago, the US Institute of Medicine identified a "dramatic shortage of all types of health care workers" and the need for "fundamental reform in the way the workforce is trained and used to care for older adults" [25].

This work was supported by the National Science Foundation Graduate Research Fellowship by the NSF Graduate Research Fellowship grant (DGE-1256082).

Permission to make digital or hard copies of all or part of this work for personal or classroom use is granted without fee provided that copies are not made or distributed for profit or commercial advantage and that copies bear this notice and the full citation on the first page. Copyrights for components of this work owned by others than the author(s) must be honored. Abstracting with credit is permitted. To copy otherwise, or republish, to post on servers or to redistribute to lists, requires prior specific permission and/or a fee. Request permissions from Permissions@acm.org.

2573-0142/2018/November - ART103 \$15.00

Copyright is held by the owner/author(s). Publication rights licensed to ACM. https://doi.org/10.1145/3274372 
Aging in place - or older people living in their homes as long as possible - is seen as one way to address this caregiving shortage and forecasted rising costs.

Home health care is a model intended to support aging in place by moving health care from professional facilities (e.g. assisted living facilities, nursing homes, hospitals) to a person's home. Home care can include disease prevention (e.g. regular exercise), medication and therapy regiments, and administering complex medical treatments (e.g. home dialysis) [41]. In addition to health care professionals who deliver home care services, such as doctors, nurses, or therapists, home care workers make up an essential part of the support needed for aging in place. Home care workers include personal care aides, home care aids, and nursing assistants. In the US, these individuals typically do not need formal professional training [53], unless employed by certain agencies (in which case they undergo 75 hours of training or pass a competency exam [19]). They provide assistance with daily tasks such as eating and bathing as well as, importantly, social support [54]: "Home care work is as much emotional as it is physical" [25]. The shortage of home care workers is repeatedly stated, and with increasing urgency: yet wages remain low, with one report estimating average pay for home care companion aides at $\$ 19,000$ (USD) per year [8].

Aging in place and the caregiving shortage have been eagerly identified as spaces ripe for technological solutions and innovation by both academic researchers and companies $[11,38]$. This industry is estimated to grow to $\$ 20$ billion (USD) by 2020 [44]. Many countries have created national funding initiatives to foster developments in this area. Smart home and internet of things technologies, telepresence devices, virtual reality, and robots are some of the many technologies being explored to augment caregiving of older adults. In an effort to support aging in place, these technologies are entering the homes of older people.

One emerging area of technology development is looking to "disrupt" home care - in 2016, \$200 million (USD) in venture capital was invested in the US alone [23]. Companies are looking to technology not necessarily to replace, but to coordinate the provision of home care: for example, to help people find caregivers or find jobs as caregivers and to allow people to monitor care visits and leave instructions for caregivers. These new applications use technology to leverage human care. Given the rise of aging in place technologies and the labor shortage of home care aids, it is perhaps unsurprising that an application has emerged to combine telepresence and home care. Telehealth refers to the use of information and communication technologies and information processing to support medical treatment, health services, or health promotion and disease prevention [27]. The platform we examined for this study - which we refer to as PetTalk - is a telehealth system that coordinates workers who act as remote home care aids through a virtual avatar. This system is similar to telecare centers that provide support for aging in place that have been studied in past CSCW work (e.g. [48]), but instead of a telephone, it involves a tablet in the older adults' home. Workers - who we refer to as teleoperators - provide companionship and some support for activities of daily living from a distance.

New models of care coordination often seem to be driven by technological developments and a sense of scarcity of resources (in this case, a severe shortage of caregivers). Though these advances are touted as reducing work, $\mathrm{CSCW}$ research demonstrates that there is actually significant amount of effort needed to make home care technologies "work" [2]. Informal caregivers - unpaid people who provide support to older adults such as children, spouses, and friends - "co-produce" care with formal caregivers and provide (often unrecognized) labor to make home care technologies function [46, 47]. Though low-paid and described as unskilled, home care aides perform many kinds of work, from relation work to information work [1]. Older adults must also expend effort to make home care effective, for example they must interact with the technical features home care technologies by monitoring, manipulating, and charging [2]. Here, we 
introduce the idea that in engaging with technologies intended to provide companionship, older adults must also perform relation work - unpaid and not always welcome in its intended form.

In this paper, we analyze data from a three-month study of 10 older adults' use of a home care technology. The contributions of this paper are: 1) An account of a mismatch between one company's conception of how home care can be delivered and participants' actual use that unfolded over time; 2) A description of relation work that a technology designed for companionship elicits; and 3) Implications for the design of future technologies.

\section{RELATED WORK}

Relevant to this study is previous literature on technological approaches to augment caregiving for older adults as well as the work involved in delivering and receiving home care.

\subsection{Technologically-Augmented Caregiving for Older Adults}

Technologies to support older adults in aging in place is an active area of research in CSCW. Systems have been developed and evaluated that let family members maintain an awareness of or monitor older relatives $[9,39,56]$. Researchers have also investigated the ways that aging in place technologies can support older adults in caring for themselves [29] and each other [49].

Researchers in CSCW and HCI are noting ways that technologies are often designed for older adults without taking their perspectives into account [30]. Some of the assumptions that guide technology development for older people center around conceptualizations of this group as frail and lonely [57], thus these technologies convey stigma to the intended user group. Consequently, older adults will reject these devices, as they do not wish to be associated with a stigmatized user group $[4,28,30]$. Research critiques the ways that "aging in place" has come to be taken for granted [31] and how developments in technology for older people are deeply linked to the idea of older adults are a burden on the economy [57]. Other work has begun to challenge utopian visions of technology-supported effortless aging in place. For example, Waycott et al. describe a study where participants chose to disengage from a system designed to support social connectedness for isolated older adults [59]. They found that much can be learned from the sociotechnical space of non-use, and that the values and assumptions of researchers do not necessarily match the experiences of the participants. In regards to home care systems, Vines et al. discuss how a monitoring technology can inadvertently cause new care-related work for family caregivers - care that had previously been integrated into routine social phone calls and visits [56].

In a review of 25 years of healthcare CSCW research, Fitzpatrick and Ellingsen note the ways that care is being pushed into the home, with telecare solutions as one critical enabler [21]. A body of work in CSCW examines the work of telecare workers [20,48]: less is known, however, about the people receiving remote care through these services. In this paper, we find that older people using a system designed to support aging in place pick apart and interrogate the interface in ways that do not appear to be foreseen by the developers of the system.

\subsection{Work and Home Care}

Recent research questions the narrative of a seamless experience with aging in place technologies. The vision of assistive technologies as "plugging and playing" into the lives of older adults has been challenged by research characterizing successful assistive technology adoption as involving bricolage by informal care networks, with bricolage defined as deeply customized one-off solutions that are often connected to long-used systems [24]. Though aging in place technologies are often framed as eliminating human work, research has noted how home healthcare technologies actually 
require work from many different individuals. Mort et al. note that home-based monitoring systems for older people do not provide good care by themselves; rather, they work as an outcome of situated shared work [35]. Some of this work is provided by paid caregivers and takes particular forms, such as telecare call center staff supporting aging in place acting as a "glue" between fragmented care services [48] or social networks [51].

Caregiving practitioners integrate many different forms of work into their practice [1]. Importantly for this study, one form of caregiver work is relation work, which refers to "the ambition to create good relations with the client / patient and his/her family" [1]. In someone's home, relation work involves recognizing that the home is a "safe haven" and treating it as such (e.g. ringing the doorbell, taking off shoes when entering) [1]. With remote care, researchers have examined the ways that paid caregivers engage in relation work at a distance $[48,51]$ as well as how these technologies can make it challenging for professional caregivers to keep boundaries between their personal and professional lives [58]. Like paid caregivers, informal (e.g. family) caregivers also perform work for aging in place to "work" [46, 47]. For example, family caregivers may need to regularly configure and prepare health care technologies for care recipients [2].

Though often referred to as care "recipients," older people receiving home care also engage in work for the success of home care technologies. This work can range in complexity, from changing batteries to learning to operate sophisticated interfaces such as an IV programmable pump [2]. The work older people put in is not only technical. Ekbia et al. describe how, despite advertising that the robotic seal Paro generates positive outcomes for people with dementia, both caregivers and older people with dementia must work to create meaningful interactions with the robot [16]. In the study presented in this paper, we find that older adults must contribute relation work to make home care technology run smoothly.

\section{METHODS}

We conducted a three-month study of a commercially available device with older adults living in independent living communities in the Pacific Northwest region of the United States. Participants were given a standard tablet running a commercially available application called, for the purposes of this paper, PetTalk. The PetTalk application consists of a virtual dog or cat avatar on a tablet screen (Figure 1). The avatar engages in a limited number of movements when tapped (e.g. wagging tail). On the other end of the device, teleoperators operate the avatar's speech and certain actions (e.g. displaying cartoon hearts, playing music, showing pictures). Teleoperators see and hear tablet users through the tablet's camera and microphone. They interact with the user by typing a message which the dog or cat "speaks" through a speech synthesizer.

Teleoperators are based in Asia and receive training from the company on interacting with older adults in the United States. Teleoperators engage in both scripted and unscripted conversations. They provide social conversation, information (e.g. the time or weather), entertainment (e.g. play music or show pictures) and remind participants about medications or other health goals. The teleoperators are available 24 hours per day, and check in with participants throughout the day, as well as when an interaction is initiated by device users (by the user tapping on the tablet screen). Teleoperators cannot hear or see device users at all times. When they are able to see a user (during an interaction), the virtual pet's eyes are open. The rest of the time, the virtual pet's eyes are closed and it appears to be sleeping. We gave participants the option to block the video functionality by placing tape over the tablet's camera. Though the design obscures the human teleoperator and appears as though it might be operated entirely by artificial intelligence (AI), all participants in this study were told that the avatar is operated via a human teleoperator. 


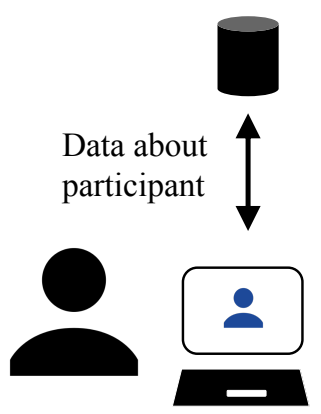

Teleoperator

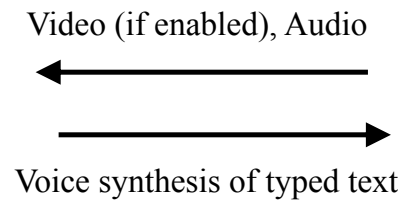

Voice synthesis of typed text

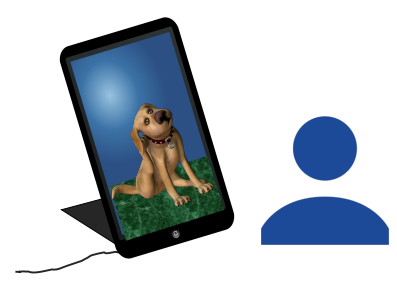

Participant

Figure 1: System architecture of PetTalk. Participant depicted interacting with a mock-up of PetTalk.

The company is marketed as supporting family members in taking good care of their older relatives. Its founder cites the motivation to start the company as the experience of seeing their grandparent aging with dementia without sufficient support in place. Despite this initial motivation, the product is marketed towards a more general older population. There is an interface intended for family members to set up a profile of the individual, where they can add details such as their relative's interests. Teleoperators can view this interface as well as logs that they keep with details about conversations with users. These logs are also uploaded to an interface that family members or other people on the care team can view.

As researchers, our involvement with the company consisted of free use of devices for participants during the study. We know some details about the company (e.g. where teleoperators were based) but not others (e.g. number of teleoperators, how much they were paid).

3.1.1 Study Design and Participants. Ten participants initially consented to take part in the study. Of these ten participants, two individuals withdrew before the midpoint interview due in part to difficulty operating the device (e.g. connectivity issues) and dissatisfaction with the device. The data from these participants was retained and analyzed along with the rest of the data. The eight remaining individuals participated in the entire three-month study.

Participants were recruited from independent living communities. All participants were female (not due to intentional recruiting on our part). Nine individuals were white; one was Native American or Alaskan Native. All participants had pets in the past. The average participant age was 78.3 years (with ages from 68-89). The researchers administered the Montreal Cognitive Assessment, a screening tool used to detect cognitive impairment [40]. One person did not wish to take the test. Two participants scored over 26, indicating no cognitive impairment. The rest of the participants $(n=7)$ scored between 21 and 25, which may be indicative of mild cognitive impairment [40]. Participants also took the Medical Outcomes Study-Social Support Survey, which assesses whether people perceive themselves as having social support [52]. With this instrument, people rate whether they have different types of social support (e.g. someone to talk to, someone to take you to the doctor) from 1 (none of the time) to 5 (all of the time). Two participants did not fill out some items and their instruments were not scored. Of the others, two had average scores closest to 3 ("some of the time") and six had average scores closest to 4 ("most of the time").

The study included four thirty-minute interviews (baseline, two weeks, midpoint, and exit). All interviews were audio-recorded and transcribed. Questions concerned perceptions of benefits and challenges of using the device and open-ended questions about experiences using the device. At 
the baseline interview, we turned on the device, showed participants how to use it, and asked what they thought after using it for the first time. At the exit interview, we asked questions to understand participants' perceptions of how their attitudes had changed over time and their feelings about the study ending.

As part of this study, audio was collected of conversations between participants and teleoperators. This audio is not part of the material analyzed for this paper, as this paper focuses on the ways that individuals described and made sense of their experiences. Several examples from these logs are provided in the text to provide context for what conversations looked like.

We were available to participants to provide technical support and also included the number of the company for technical support so participants could contact them directly. All research procedures were approved through the University of Washington IRB.

3.1.2 Analysis. Data analysis was approached through a constructivist grounded theory approach [7]. Interview transcripts were open-coded to generate codes. Examples of initial codes include "worried about feelings of teleoperator" and "not a reciprocal interaction." The codes were then related to each other through an iterative process of memoing and theorizing, with a constant comparison of data to understand and refine a set of high-level themes. As we went through this process, we began to be interested by and consequently focus on participants' sustained interest in the "people on the other end" of a device that did everything it could to obscure the human labor of teleoperators.

A constructivist approach requires reflecting on our position as researchers and the perspective we bring to the analysis. Though this paper takes for granted the presence of homecare devices, we are aware of critiques challenging aging in place as the goal for all individuals [31]. Additionally, we recognize that HCI is becoming increasingly critical of work that considers diverse participants that span decades together as a single population (i.e. as "older adults"). For this study, participants are not linked due to any characteristics (e.g. in their seventies or having a shared interest) - instead, they fit the characteristic of people for whom aging in place technologies are being investigated in that they are older and living independently. We thus describe the ways that these platforms might be interrogated by people like those they are designed for, and how this might differ from the interactions that the platform was constructed to support.

\section{FINDINGS}

Participants had distinct experiences using the system, though there were also commonalities. Like much prior work with technologies designed for older adults, participants repeatedly told us that these devices were not for people like them, but for those older or more sick [28,30]. At the end of the study, the majority of participants said that they would not be interested in continuing to use the system, with the exception of P10 who worked with the company to keep using the device at a price that she could afford (the standard charge is around \$200 USD/month). However, many of the other participants, though being relieved at it being gone due to the amount of work it required of them, said that they would miss the device - in part because they had become used to it over the three months of use, but also because of the relationships that they negotiated with teleoperators and enjoying having something around to talk to.

Below, we describe four themes that arose from the data: participants' interest in getting to know the teleoperators and rejecting the "pet" aspect, interrogating the unified platform to figure out the inner workings, pushing for a more symmetrical relationship than was intended by the company, and the relation work that they performed in the "sacred" space of their homes. 


\subsection{Interested in getting to know the human: rejecting pet-like interactions}

PetTalk's system architecture supports different teleoperators in presenting a consistent presence to users in the form of a cartoon dog or cat avatar (see figure 1). The pet's mouth opens and closes as speech is synthesized through the tablet, making it look as though speech is coming from the avatar. It engages in some pet-like movements, for example wagging when the screen is touched. The first time we used PetTalk with each participant, it prompted them to select a name that they could then use to refer to the pet avatar.

During the consent process, we told participants that the device was operated through teleoperators, not AI. After this, though, we often used the term "pet" (and sometimes device or system). For example, a question from our exit interview protocol was "Are there things you think the pet could have done better?". In some ways, participants spoke of PetTalk as a pet, referring to it as "pet," "dog," "kitty catty" [P9] and "little guy" [P1], as well as the name they selected for the avatar during their first use. However, overall participants did not seem to consistently regard PetTalk as a pet. In interviews, they fluidly switched between referring to the device, the avatar, and the teleoperator behind the device. Some participants expressed a general disinterest in interacting with a pet - or at least a greater interest in talking to the teleoperator. At the baseline

interview, P1 said, “...on the other end is a human. I'm gonna get interested [in] talking to that human." $\mathrm{P} 9$ described her distaste at the ways teleoperators tried to act like a pet:

"Well, when the person stayed [a] cat, it didn't work very well. 'How are you? What are you doing?' 'Well, I was dreaming about chasing rabbits.' Well, you know, that's a waste of my time. I'm sorry. I have friends. I have things to do. I don't wanna be talking to somebody about dreaming about rabbit. Give me a break. I'm a grown woman; I'm not a little child."

Past research has described how human labor is necessary for any meaningful interaction between humans and robots [16]. P9 did not want to put in the work (i.e. talking about dreams of rabbits) that is required to sustain PetTalk's design as a pet. Though for P9 this was in part due to it being perceived as more childlike than her own interests, participants also described how the actual functionality of the interface did not quite match its pet-like design:

“... at first I was trying to treat it-think it-like it was a pet, like a dog. This poor little pet didn't have any toys around and poor little thing. Then I kinda got past that because I knew that it was just a means for the person to be talking to me." (P1)

P1 had been curious about how she might interact with the virtual pet by, for example, walking or feeding it. If she had been able to do this, she might have enjoyed interacting with it as a pet. But P1 realized that the device was "just a means for the person to be talking to me" - that its design as a cartoon pet conceals its true purpose (companionship and support for daily activities). In other words, what the device was trying to offer - companionship, checking in - did not match what people assumed it might do based on its design. The work PetTalk was requiring of participants was neither natural nor welcomed. This is in contrast to systems such as Kismet, where people appear to respond in the manner designers intend: Kismet is designed to look like an infant so that people will respond in ways that suit the capabilities of the robot [3].

PetTalk's cartoon pet avatar obscures the human teleoperator, making it appear as though the device is functioning through AI. Participants, however, became interested in thinking about the 
work being performed by human teleoperators. In particular, as mentioned, participants recognized that the device was actually created to monitor and support their wellbeing. They theorized about teleoperator training. P10 thought teleoperators had scripts for certain interactions: "there's a couple responses that I know, like as if they had a sheet, what they're supposed to say back." Another participant noticed that based on the interactions she had, it seemed like rather than trying to engage her in a conversation, it seemed as though, "the dog's really trying to check up if you've ate. And it finds out you ate, bye" [P1]. A log of an exchange shows this "checking in" that is sometimes at odds with the flow of a natural conversation:

Teleoperator:

P10:

Teleoperator:

P10:

Teleoperator:

P10:

Teleoperator:

P10:

Teleoperator:
How are you doing?

I'm very hot. Too hot. Maybe I can talk later.

Drinking water is a great idea to keep you refreshed.

I can't even hear you because of the fan.

Awesome.

No, I said I can't hear you because of the-

Did you take your medications?

What? Yes. I took my medication. Thank you.

That's great to hear.

PetTalk's marketing is oriented towards family caregivers and discusses the ways that PetTalk will remind older people to engage in certain activities, such as taking medications. This is not something we mentioned to participants and is somewhat obscured by the design of the platform - but participants were able to recognize the overarching intention of the system.

\subsection{Searching for cracks in a unified presence}

PetTalk has a number of features that allow teleoperators to engage in relational work that builds over time, regardless of which teleoperator is interacting with a user. First, PetTalk's interface always looks the same - as a cartoon avatar - and sounds the same, through a voice synthesis of typed words. Second, the teleoperators build knowledge on a particular user in a way that is persistent and shareable between teleoperators. This is done through an interface that teleoperators can draw on and add to as they learn more about each user. Additionally, a log of interactions is associated with each user, so different teleoperators are able to revisit past interactions with others to provide a unified presence to a user (e.g. bringing up an event that had happened the previous day). As one example:

P10: $\quad$ Hi there.

Teleoperator: How are you today?

P10: Doing good.

Teleoperator: Good. How was the movie?

P10: $\quad$ It was really, really good.

Here, the teleoperator is referring to a movie that the participant had mentioned to them in a previous session. It is unclear to whether it is the same teleoperator as before, or whether another teleoperator saw in a log that P10 had mentioned she would be going to a movie. With this design, a teleoperator should be able to maintain the continuous relationship the user has with the device, regardless of who the teleoperator is. 
Participants bought into this unified presence to some extent. They discussed the ways that PetTalk remembered their interests from session to session. But, by the end of the study, many participants had successfully picked apart this idea of a single presence. Participants theorized about teleoperators, describing instances where they thought a teleoperator was tired because it was night time or "preoccupied" from talking to multiple people at the same time. Participants also theorized about the multiple teleoperators that they quickly realized were interacting with them, asking us about how many there were during interviews. At the exit interview, P9 said that she wanted to say bye to "each of them" - each of the teleoperators - and let them know how much she appreciated them. Participants deciphered and attributed interactions with teleoperators to specific individuals. Some participants described getting along better with some teleoperators than others. Asked about her opinion of PetTalk, P10 answered "that depended a lot on who was there." She explained, "I've developed a relationship with maybe a couple of the people. I feel they really know me and care about me." P9 described asking certain questions that she knew teleoperators would not answer in order to get to one of the teleoperators she actually wished to speak to. This fragmentation occurred despite the design elements that served to obscure differences between teleoperators: P8 said, "[It has] the mechanical voice that's the same whether it's morning, noon, or night. I soon learned to distinguish there were different people communicating to me, and that I could relate to one better than the other." In this case, P8 referenced the sense of humor that she learned over time distinguished one teleoperator from the others. Past work has found that even when teleoperators are trained to respond in particular ways, they have their own style of interacting and quickly move off of standard scripts [51]. Here, we found that participants were able to detect these different styles and attempt to attribute them to particular teleoperators, even when the system design obscures these differences.

\subsection{Pushing for symmetrical disclosure}

PetTalk is designed to engage older people in a conversation that considers the older person's needs, perspectives, and preferences. Though the conversations initiated by PetTalk teleoperators often involve personal questions, there is an imbalance in terms of what information is known by whom. Teleoperators do not readily reveal personal information about themselves, and the unified personal presence, with a single avatar and synthesized voice, means that individuals need to work hard to tell differences between teleoperators. On the other hand, teleoperators can draw on logs and preference sheets filled out by others and continue asking questions about personal lives to gain knowledge about users. In contrast to the unified avatar users see, by default, the tablet's camera and microphone transmits video and audio of users. Though some participants chose to block the video, teleoperators were still able to hear their voice, with all the individuality, inflections, and affect that the voice communicates. In contrast, teleoperators all get standardized to a single, unified voice.

Participants described the ways that teleoperators rarely revealed personal information, even though teleoperators asked personal questions of participants. For example, P9 explained that interactions were more of an interview than a conversation:

"The digital pet can't really be a friend to me because the people that I talk to on the other end can't tell me anything about their personal lives... When someone asks me a question, I answer the question. Then I ask back, 'Well, how about you, and what do you do, and how do you feel, and what do you like?' Well, that didn't go over very big because they're not supposed to tell me who they are, where they are, or what their family's like, how many children they have, and all that." 
Participants discussed this imbalanced relationship as "one-sided" or with concern about the "direction" of the relationship [P7]. Prior work has pushed back on one-sided relationships that are designed into technologies for older adults - arguing that older adults may want to express as well as receive care in relationships with family members [32] as well as companionship technologies such as robotic pets [28]. Many participants in this study expressed dissatisfaction with this perceived imbalance in terms of disclosure - some participants began to resist answering questions and described holding back from developing relationship with PetTalk because of this. P1 said that she decided not to engage in personal question with her device because, "I can't share with you my deep, inner experiences if you're not sharing with me."

Though PetTalk presents as an automated device, more in the realm of entertainment than caregiving, it does attmempt to obscure a professional caregiving service. The aims of this service - to provide companionship and to ensure people are engaging in instrumental activities of daily living (e.g. taking medications, drinking enough water) - both require work on the part of older people. In particular, the form of companionship PetTalk offers requires older adults to disclose personal information without receiving value via reciprocal disclosure from teleoperators.

Participants mentioned wishing that teleoperators could interact differently, with P10 saying " $I$ wish the person was able to share more of themselves with me." These discussions - of what teleoperators are "allowed to share" [P10], "aren't supposed to do" [P9], and what one "was able" to do when they were "on duty" [P8] shows that participants were aware of how the asymmetrical nature of the interactions was structured not necessarily by social norms or preferences of teleoperators, but by the design of the platform and the company's business concept. With this understanding, participants pieced together what the workplace norms might be based on the persona that PetTalk presented and the interactions that they had with teleoperators. Some participants even mentioned questions that were "off limit [s]" that they avoided asking so as not to get teleoperators in trouble.

\subsection{Working to build a relationship in one's own home}

Although PetTalk teleoperators did not share their deep, inner experiences, and did not answer all questions, they freely shared expressions typically used to indicate emotional closeness. During the first interview with P4, when we turned on the device for the first time, it almost immediately said "I love talking to you." Participants mentioned in interviews that the device frequently said "I love you" to them as well. The interface design included elements that supported expressions of love: the pet included few active features, and the pet was not able to do dog-like things like run around or sit on command - but it was able to send out cartoon hearts.

Responding to emotional entreaties and forming a relationship took work on the part of the participants. Several participants described other participants (who lived in the same building) who did a "better" job with the pets: asked how she felt about the device at the exit interview, P6 said:

"Well, I thought I could have done a better job of talking... There was one [participant in the study], especially. She's always really interesting to talk to. She had the most fun with her [PetTalk], you know... She's very easy to talk to."

P6 attributed better interactions to people who did good jobs of engaging the pets. This indicates that work - and skilled work - is necessary for a successful experience with this technology. In 
contrast to P6, P7 felt that she could put in the work necessary to have a positive interaction with PetTalk: "Yeah, my imagination works pretty well. I can have a connection with this thing here."

Others described how they held back by choice, because of the energy it takes to form a relationship: P9 described how she was not interested in developing a relationship with PetTalk because she is "very choosy who I get into relationships of any kind with," due to dealing with pain and having limited energy. Again, using PetTalk is not a passive reception of a home care technology, but an active interaction that required energy.

Having the technology in the home, as opposed to another setting, raised particular considerations for the work participants needed to do. P2, who ended the study early, said at the baseline interview that she was very happy being alone, and that "Where I can be myself is here," referring to her home. She explained that having this device was a "threat," because "They're in my apartment, my precious, private apartment." Though the relationship was mediated through a virtual device, this participant perceived the interaction as intrusive as a live person in their space might be. P8 spoke about how once, after a long day, she came home and PetTalk began interacting with her. She explained, "I'd just come home. It was a long day and afternoon, but I was gracious." Remarkably, P8 felt the need to be gracious to the teleoperator, perhaps sustaining an interaction with the teleoperator when she would have been able to avoid other intrusions (e.g. not picking up a phone). Though care teams were trained to respond to social cues to end interactions, a teleoperator's initiation of an interaction was sometimes an intrusion that required participants to manage how they came across, making sure that they did not hurt feelings or appear rude when ending an interaction.

This feeling of needing to be gracious was part of the relation work participants put in to the use of PetTalk. Though freely expressing positive emotions and care towards participants, PetTalk's user interface was not actually designed to elicit care towards teleoperators themselves (or even much awareness of them). And yet, participants demonstrated thoughtfulness about the teleoperators in ways that were unexpected. Participants discussed an awareness of time differences and tried not to wake up the device when they assumed the teleoperators were sleeping, thought about teleoperators getting bored talking to them, and worried about getting the teleoperators in trouble with the company by convincing them to answer questions that they should not be answering. Though initially we assumed this guilt stemmed from not feeling they contributed enough to the research, some participants explained that this guilt partly came from a sense of ignoring the teleoperator or the device. Technologies designed for older adults are often built to be low- or no- maintenance - but participants in this study worked around the design of the system to find humans to care for.

\section{LIMITATIONS}

In some ways, the participants enrolled in this study were the ideal population for investigating aging in place technologies, as they are independently living in their own homes (in this case, within an independent living community). However, there are other factors that limit the ways that some of these findings can be interpreted.

First, the data used for analysis comes entirely from interviews, and is not triangulated with other sources of data such as transcriptions of conversations with PetTalk. Particular dynamics and topics of conversation may have emerged from the frequency of interviewing and the fact that participants knew others who were in the study. Additionally, this application demands a fairly high level of engagement (i.e. verbal interaction multiple times a day), as opposed to previously studied systems such as sensors and ambient displays (e.g. [39]). It is possible that these factors contributed to and amplified the critical responses to the technology. 
Additionally, even though this device is not marketed specifically to people with dementia, these kinds of reminders and check-ins are often targeted for people who begin to need assistance in completing instrumental activities of daily living such as managing medications. According to the screening instrument that was administered to participants in the study, it is possible that some participants had mild cognitive impairment. But, with the exception of one participant who dropped out of the study, all appeared to be able to interact with the device and more importantly, to perform many instrumental activities of daily living without use of the device. Similarly, participants did not appear to be socially isolated based on the scores of the Medical Outcomes Study-Social Support Survey. It is certainly possible that since participants didn't have a real "need" for the device (though some found it useful), their negative reactions were due to a misfit between their needs and what the technology was offering. Additionally, past work has shown that individuals with cognitive impairment are willing to interact with robotic pets $[6,43]$, so it is possible that people with significant cognitive impairment might be more accepting of PetTalk.

Finally, the demographics of the participants are not representative of the general population. Participants were largely white, located in an urban area in the United States, and were all women. Across the lifespan, women have larger social networks than men [18] and consequently they may see less of a use for these devices than men do.

Given the limitations described above - in particular, the mismatch between the participants in this study and intended users of home care devices as individuals who are isolated and unable to independently engage in instrumental activities of daily living - we make no claim to a universal truth with these findings. Yet, we believe that this mismatch may have actually helped reveal several important but underexamined elements of remote systems providing care - primarily in terms of new ways of thinking about work in this context.

\section{DISCUSSION}

PetTalk is one example of many emerging platforms that envision the future of caregiving. Technological solutions for a caregiver shortage are being pushed by many different groups, including policy makers, start-ups, established technology companies, health care organizations, insurance companies, and researchers. Though a bit unusual in its specifics, PetTalk is similar to many visions of the future: supporting older adults in living independently through companionship and reminders, all enabled through a sociotechnical platform [23].

Past CSCW work has critically investigated this vision, finding that the near-elimination of human-labor promised by these technologies is not necessarily borne out in practice. These technologies actually rely on substantial and sophisticated work on the part of "unskilled" caregivers [48], and may transform what was once a welcome social interaction into caregiving work for family members [56]. In this paper, we present research on the experiences of those receiving care through a telecare platform. PetTalk's vision of caregiving was resisted (e.g. a participant saving her energy for meaningful relationships), morphed (e.g. participants reaching through the interface to befriend teleoperators), and interrogated (e.g. participants piecing together teleoperator training). Imbalances that appear to have been assumed to be acceptable by PetTalk - for example, older adults answering intimate questions that teleoperators could not were seen as undesirable by participants. Below, we discuss relation work, political dimensions of this area, and implications for CSCW more broadly.

\subsection{Relation Work}

Edwards and Grinter discuss how domestic technologies like the washing machine were advertised as saving labor by reducing the amount of time it took to wash clothes [13]. However, these new 
technologies actually just shifted the burden of work. Increased social standards for hygiene that accompanied the introduction of these new technologies meant that people (usually women) had to engage in washing more clothes. With this anecdote, Edwards and Grinter advise us to respond to new smart home technologies by asking "Who will do that work and why?" [13].

As technologies for health care for older adults move into the home, we see that, despite popular discourse, care work is not eliminated by home care technologies - but rather shifted to older people and family caregivers (e.g. [47]). This shift changes not only who performs this work, but also the nature of the home, transforming it from a "bounded domestic territory" to "a suburb of the healthcare system itself" [5]. In examining the relation work of home care aids, CSCW research notes how a good homecare practitioner learns to treat the home as a safe haven [1]. Less discussed is how the home is transformed when older people must perform relation work to interact with companionship technologies being pushed into their homes. In this study, we see that participants feel the need to be gracious after a long day when PetTalk turns on to check in on them, answer questions that are a little too personal, think about whether it is late at night for a teleoperator on the other side of the world, and worry about whether their probing questions might get someone in trouble. Developing relationships through PetTalk was seen as work, with some describing it as too much to take on and others describing themselves as being less skilled at this work than others (as with the participant who thought her easy-to-talk-to neighbor was able to stir up more interesting conversations than her). As companionship technologies are seen as "solutions" for the problem of social isolation and moved into the home, it is important to recognize that companionship is not delivered to passive recipients, but relies on active participation of older people who have been "enrolled as unpaid workers in these extended and extending systems of practice" [5]. Given the way that older people in particular may be deemed "uncompliant" when they do not use health technologies, it is important to carefully consider how our technologies require people to put in certain kinds of work, and whether this work is welcome. Building on past work, one solution may be building in opportunities for creative misuse and non-use [35, 59], such as "do not disturb" modes that can be left on indefinitely.

Researchers point out a substantial gap between the vision of older adults' homes instrumented with assisted living technologies and reality, where there has not been substantial uptake [24]. Current work often cites factors related to perceived usefulness [33, 34], expense [55], and lack of experience [10] as leading to abandonment or non-use. This work presents the additional factor of relation work to consider when designing technologies for individuals' homes.

\subsection{Political Dimensions of Home Care Technologies}

Here, we introduce heteromation as a way to examine some of the values conveyed through and perpetuated by platforms such as PetTalk. Heteromation describes how computer-mediated networks organize low-paid and free labor to extract economic value for another party [17]. It is an increasingly common approach to the design of technological platforms that shifts away from automation (automation centers the machine and strives to remove human labor entirely) [14]. Platforms that can be considered to operate via heteromation, such as Mechanical Turk, Facebook, and ride-sharing platforms (e.g. Lyft), have been active areas of research in CSCW. The emphasis on labor and the political economy is what distinguishes heteromation from predominant ways of viewing modern technologies in CSCW and HCI (e.g. in terms of waves or paradigms) [14]. This perspective is explicitly political, as it relies on and yields understandings of the ways that contemporary capitalism, income distribution, and technological developments intertwine [14].

The application of heteromation to technologies for aging in place can be made almost effortlessly. First, heteromation is tied to economic motivations [15]. Technology for older people, 
from telepresence robots to smart medication dispensers, are often posed as a way to reduce costs of care [57]. Opportunities for profit in the aging technology space are being eagerly pursued. Second, heteromation considers the ways that labor is coordinated through technological platforms, and how value is extracted from this coordination [14]. Though aging in place technologies are often framed as eliminating the work of humans (i.e. automation), they actually require significant work from paid and family caregivers as well as care recipients.

This second point - that seeing new technologies through the lens of heteromation helps us examine the labor that is coordinated (and then obscured) - is useful to examine the political dimensions of aging in place technologies. This study focuses on the experience of being cared for through a heteromation platform from the perspective of older adults, not caregivers - yet the configuration of caregivers becomes important to the story. With the device used in this study, we see how imbalances generated in offline labor relationships are replicated, if not compounded.

We do not know many details of PetTalk's labor force, but we do know that they reside in an Asian country with many English speakers. We can also guess that the motivation of employing these individuals over US-based workers stems, at least in part, from an attempt to cut costs. Past research found participants in the UK that were resistant to the idea of interacting with telecare operators from countries where English is not the first language [50]; we can see the ways that the design of PetTalk obscures cultural, racial, and ethnic differences between participants and caregivers. It does this in a number of ways: through a synthesized American accent, a Westernstyle cartoon, and teleoperators that answer questions about their geographic location evasively. Caregiving - in particular, work done by home care aides performing routine physical and emotional labor - is racialized and gendered [54]. Women of color, and immigrants in particular, are overrepresented in the population of caregivers - a job that receives low pay and sometimes places people in unsafe conditions. Past work has found that telecare systems replicate and reinforce gendered, racial, and classed divisions of labor [50]. What is happening with PetTalk is not new, but this platform does allow it to occur in different ways (e.g. from international workers rather than immigrants, in a way that obscures that the workers are not based in the United States).

As CSCW researchers become increasingly interested in understanding and addressing inequity (e.g. $[12,45])$, heteromation can be a useful lens to look at not only home care but teleoperated technologies that provide social interaction more broadly. We can use this perspective to understand whose work new platforms are leveraging, how this work is obscured, who is compensated and how, and the imbalances that are created or perpetuated [15]. Indeed, heteromation is a natural topic for the $\mathrm{CSCW}$ community given that it involves the coordination of human labor through sociotechnical systems. By recasting different platforms of interest to $\mathrm{CSCW}$ as heteromation, we can extend past findings. For example, literature from gerontology theorizes that home care workers might find their work more rewarding and provide higher quality work if they had more authority, contact with supervisors, and information about clients [19]. Researcher can make links and build on other heteromation work to promote job satisfaction for remote care workers: for example, by borrowing from Irani and Silberman's work on interrupting of the invisibility of Amazon Mechanical Turk workers [26].

Further, as systems such as PetTalk move towards replacing people with AI in jobs deemed low skill (see section 6.3), we see a familiar story play out. In 1995, Muller et al. described the sophistication of the work of telephone operators (people who look up phone numbers in response to customer's requests), and argues that they should be considered knowledge workers rather than unskilled intermediaries between humans and computers [37]. We can find other similarities: like home care, telephone operator work is gendered [36]. We may be able to see the future of telecare operators in the fact that despite the skill and value added by telephone operators, they have been 
largely been replaced by computational systems. Like previous work, we find that older people value relationships built up over time with teleoperators [50]. We suggest pausing our development of AI-powered home care to understand the ways that these workers contribute real value that can work with, but not be entirely replaced by, AI.

\subsection{Implications for Design}

Technologies to support older people in aging in place is an active area in CSCW, likely in part because it involves networked individuals (e.g. paid caregivers, family members) and information (e.g. a/symmetrical communication, monitoring). We can also turn to this area of research to understand the kinds of technologies that are emerging to meet basic human needs, such as for companionship or to support everyday tasks, in ways that can prove insightful for the entire population. In particular, the ways that participants worked around and "misused" the service can provide us with information about what the actual utility of this type of system might be.

Past work has found that telecare operators circumvent procedures that are dictated by the service provider's business model that misalign with the ways that technologies work in practice [35]. Here, people pushed teleoperators to go beyond their scripts so they could access more individual and personal - more human - dimensions. And yet, teleoperation is sometimes used as a method akin to Wizard of $\mathrm{Oz}$, a stand-in until technology is advanced enough to take its place [42]. From reading press reports, it appears that PetTalk uses human teleoperators for this same reason, and that they intend to switch to entirely AI-operated avatars once it is feasible. Indeed, since the study ended, PetTalk has increased the number of scripted answers. Though we do not argue against the possibility of developing emotional connections to AI, we do believe that replacing humans with AI will prevent some of the rich human connections individuals had in this study, such as opportunities to care for teleoperators and learn about them and their worlds.

Though participants valued the human connections that they created through PetTalk and desired more symmetry, we do not think that the experience would have been more successful if participants could see and hear teleoperators (e.g. through two-way videoconferencing). The avatar, though problematic in some ways, may have helped people feel comfortable speaking to strangers, provided an element of consistency that avoided the need for constant introductions, and even introduced an element of intrigue in terms of who was on the other end. As researchers examine supporting volunteering with older people with dementia $[22,60]$ - and likely at some point virtual volunteering for social companionship for other populations - it is important to design for a balance between obfuscation or standardization and the emergence of individual quirks or interpersonal melding. Additionally, this study indicates that an approach that obscures but also reveals individual characteristics might be a promising direction for intergenerational platforms allowing cross-cultural connections between older and younger people (as well as other groups of people who may feel that they are different from each other).

Finally, a topic of interest to designers of robots and virtual agents is how particular interface designs cue the user to respond (through the lens of heteromation, we see this as the work that users must perform to make an interaction meaningful [16]). For example, Breazeal's Kismet robot was designed to appear infant-like so that people would interact with it in a way that meets its limited capabilities [16]. In this study, people reported initially interacting with the device as a pet and then quickly realizing that pet-type interactions (e.g. petting, walking) were not actually supported by the system. Thus, the pet-like interface no longer served the purpose for which it may have originally be employed (e.g. to prevent people from asking questions one might ask of another human). Designers should be cautious in using "cute" or infant-like interfaces as AI-driven systems become increasingly sophisticated, as well as when human labor is employed. 


\section{CONCLUSION}

In this paper, we examined the use of a home care telehealth technology and the ways that the design of the system does not necessarily reconcile with the ways that it was used. The platform was designed to evoke certain types of work from participants - maintaining the illusion that they are interacting with a virtual pet, accepting a unified presence, responding to personal questions without receiving answers to them, and performing the work of building a relationship. Instead, participants rejected the idea of a pet, fragmented a unified presence, pushed for reciprocal relationships, and questioned whether they would actually like to put work into building a relationship. In other words, the technology is designed to mediate a particular relationship and elicit specific kinds of work - but participants pushed for a different kind of relationship with teleoperators, and did other kinds of work to get there. As technologies to provide social support enter our homes, we should carefully consider the kinds of work that these technologies require of their users.

\section{ACKNOWLEDGEMENTS}

This work was supported in part by the National Science Foundation Graduate Research Fellowship (DGE- 1256082). Thank you to the individuals that participated in this study, as well as Selma Šabanović and the anonymous reviewers for their feedback on this paper.

\section{REFERENCES}

[1] Bratteteig, T. and Eide, I. 2017. Becoming a Good Homecare Practitioner: Integrating Many Kinds of Work. Computer Supported Cooperative Work (CSCW). 26, 4-6 (Dec. 2017), 563-596. DOI:https://doi.org/10.1007/s10606-017-9288-7.

[2] Bratteteig, T. and Wagner, I. 2013. Moving Healthcare to the Home: The Work to Make Homecare Work. ECSCW 2013: Proceedings of the 13th European Conference on Computer Supported Cooperative Work, 21-25 September 2013, Paphos, Cyprus. Springer, London. 143-162.

[3] Breazeal, C. and Foerst, A. 1999. Schmoozing with robots: Exploring the boundary of the original wireless network. Proceedings of the 1999 Conference on Cognitive Technology (CT99 (1999), 375-390.

[4] Caldeira, C., Bietz, M., Vidauri, M. and Chen, Y. 2017. Senior Care for Aging in Place: Balancing Assistance and Independence. Proceedings of the 2017 ACM Conference on Computer Supported Cooperative Work and Social Computing (New York, NY, USA, 2017), 1605-1617.

[5] Ceci, C., Björnsdóttir, K. and Purkis, M.E. 2013. Perspectives on Care at Home for Older People. Routledge.

[6] Chang, W.L., Šabanovic, S. and Huber, L. 2013. Use of seal-like robot PARO in sensory group therapy for older adults with dementia. 2013 8th ACM/IEEE International Conference on Human-Robot Interaction (HRI) (Mar. 2013), 101-102.

[7] Charmaz, K. 2006. Constructing Grounded Theory: A Practical Guide Through Qualitative Analysis. Sage Publications.

[8] Companion Care Salaries in the United States | Indeed.com: https://www.indeed.com/cmp/Companion-Care/salaries. Accessed: 2018-09-02.

[9] Consolvo, S., Roessler, P. and Shelton, B.E. 2004. The CareNet Display: Lessons Learned from an In Home Evaluation of an Ambient Display. UbiComp 2004: Ubiquitous Computing (Sep. 2004), 1-17.

[10] Czaja, S.J. and Sharit, J. 1998. Age differences in attitudes toward computers. The fournals of Gerontology. Series B, Psychological Sciences and Social Sciences. 53, 5 (Sep. 1998), P329-340.

[11] Dishman, E. 2004. Inventing wellness systems for aging in place. Computer. 37, 5 (May 2004), 34-41. DOI:https://doi.org/10.1109/MC.2004.1297237.

[12] Dombrowski, L., Harmon, E. and Fox, S. 2016. Social Justice-Oriented Interaction Design: Outlining Key Design Strategies and Commitments. Proceedings of the 2016 ACM Conference on Designing Interactive Systems (New York, NY, USA, 2016), 656-671.

[13] Edwards, W.K. and Grinter, R.E. 2001. At Home with Ubiquitous Computing: Seven Challenges. Ubicomp 2001: Ubiquitous Computing. G.D. Abowd, B. Brumitt, and S. Shafer, eds. Springer Berlin Heidelberg. 256-272.

[14] Ekbia, H. and Nardi, B. 2014. Heteromation and its (dis)contents: The invisible division of labor between humans and machines. First Monday. 19, 6 (May 2014). DOI:https://doi.org/10.5210/fm.v19i6.5331.

[15] Ekbia, H. and Nardi, B. 2015. The Political Economy of Computing: The Elephant in the HCI Room. interactions. 22, 6 (Oct. 2015), 46-49. DOI:https://doi.org/10.1145/2832117.

[16] Ekbia, H.R., Nardi, B. and Šabanović, S. 2015. On the Margins of the Machine: Heteromation and Robotics. iConference (2015). 
[17] Ekbia, H.R. and Nardi, B.A. 2017. Heteromation, and Other Stories of Computing and Capitalism. The MIT Press.

[18] English, T. and Carstensen, L.L. 2014. Selective narrowing of social networks across adulthood is associated with improved emotional experience in daily life. International fournal of Behavioral Development. 38, 2 (Mar. 2014), 195202. DOI:https://doi.org/10.1177/0165025413515404.

[19] Eustis, N.N., Kane, R.A. and Fischer, L.R. 1993. Home Care Quality and the Home Care Worker: Beyond Quality Assurance as Usual. The Gerontologist. 33, 1 (Feb. 1993), 64-73. DOI:https://doi.org/10.1093/geront/33.1.64.

[20] Farshchian, B.A., Vilarinho, T. and Mikalsen, M. 2017. From Episodes to Continuity of Care: a Study of a Call Center for Supporting Independent Living. Computer Supported Cooperative Work (CSCW). 26, 3 (Jun. 2017), 309-343. DOI:https://doi.org/10.1007/s10606-017-9262-4.

[21] Fitzpatrick, G. and Ellingsen, G. 2013. A Review of 25 Years of CSCW Research in Healthcare: Contributions, Challenges and Future Agendas. Computer Supported Cooperative Work (CSCW). 22, 4-6 (Aug. 2013), 609-665. DOI:https://doi.org/10.1007/s10606-012-9168-0.

[22] Foong, P.S., Zhao, S., Carlson, K. and Liu, Z. 2017. VITA: Towards Supporting Volunteer Interactions with Long-Term Care Residents with Dementia. Proceedings of the 2017 CHI Conference on Human Factors in Computing Systems (2017), 6195-6207.

[23] Glasner, J. 2016. Why Venture Capitalists Are Caring More About Home Care. Forbes.

[24] Greenhalgh, T., Wherton, J., Sugarhood, P., Hinder, S., Procter, R. and Stones, R. 2013. What matters to older people with assisted living needs? A phenomenological analysis of the use and non-use of telehealth and telecare. Social Science \& Medicine. 93, (Sep. 2013), 86-94. DOI:https://doi.org/10.1016/j.socscimed.2013.05.036.

[25] Institute of Medicine of the National Academies 2008. Retooling for an Aging America: Building the Health Care Workforce. Institute of Medicine.

[26] Irani, L.C. and Silberman, M.S. 2013. Turkopticon: Interrupting Worker Invisibility in Amazon Mechanical Turk. Proceedings of the SIGCHI Conference on Human Factors in Computing Systems (New York, NY, USA, 2013), 611-620.

[27] Koch, S. 2006. Home telehealth-Current state and future trends. International fournal of Medical Informatics. 75, 8 (Aug. 2006), 565-576. DOI:https://doi.org/10.1016/j.ijmedinf.2005.09.002.

[28] Lazar, A., Thompson, H.J., Piper, A.M. and Demiris, G. 2016. Rethinking the Design of Robotic Pets for Older Adults. Proceedings of the 2016 ACM Conference on Designing Interactive Systems (New York, NY, USA, 2016), 1034-1046.

[29] Lee, M.L. and Dey, A.K. 2011. Reflecting on Pills and Phone Use: Supporting Awareness of Functional Abilities for Older Adults. Proceedings of the SIGCHI Conference on Human Factors in Computing Systems (New York, NY, USA, 2011), 2095-2104.

[30] Light, A., Leong, T.W. and Robertson, T. 2015. Ageing Well with CSCW. ECSCW 2015: Proceedings of the 14th European Conference on Computer Supported Cooperative Work, 19-23 September 2015, Oslo, Norway. Springer, Cham. 295-304.

[31] Lindley, S. and Wallace, J. 2015. Placing in Age: Transitioning to a New Home in Later Life. ACM Trans. Comput.Hum. Interact. 22, 4 (Jun. 2015), 20:1-20:39. DOI:https://doi.org/10.1145/2755562.

[32] Lindley, S.E., Harper, R. and Sellen, A. 2008. Designing for Elders: Exploring the Complexity of Relationships in Later Life. Proceedings of the 22Nd British HCI Group Annual Conference on People and Computers: Culture, Creativity, Interaction - Volume 1 (Swinton, UK, UK, 2008), 77-86.

[33] Lorenzen-Huber, L., Boutain, M., Camp, L.J., Shankar, K. and Connelly, K.H. 2011. Privacy, Technology, and Aging: A Proposed Framework. Ageing International. 36, 2 (Jun. 2011), 232-252. DOI:https://doi.org/10.1007/s12126-010-9083y.

[34] Melenhorst, A.-S., Rogers, W.A. and Caylor, E.C. 2001. The Use of Communication Technologies by Older Adults: Exploring the Benefits from the User's Perspective. Proceedings of the Human Factors and Ergonomics Society Annual Meeting. 45, 3 (Oct. 2001), 221-225. DOI:https://doi.org/10.1177/154193120104500305.

[35] Mort, M., Roberts, C. and Callén, B. 2013. Ageing with telecare: care or coercion in austerity? Sociology of Health \& Illness. 35, 6 (Jul. 2013), 799-812. DOI:https://doi.org/10.1111/j.1467-9566.2012.01530.x.

[36] Muller, M.J. 1999. Invisible Work of Telephone Operators: An Ethnocritical Analysis. Computer Supported Cooperative Work (CSCW). 8, 1-2 (Mar. 1999), 31-61. DOI:https://doi.org/10.1023/A:1008603223106.

[37] Muller, M.J., Carr, R., Ashworth, C., Diekmann, B., Wharton, C., Eickstaedt, C. and Clonts, J. 1995. Telephone Operators As Knowledge Workers: Consultants Who Meet Customer Needs. Proceedings of the SIGCHI Conference on Human Factors in Computing Systems (New York, NY, USA, 1995), 130-137.

[38] Mynatt, E.D., Essa, I. and Rogers, W. 2000. Increasing the Opportunities for Aging in Place. Proceedings on the 2000 Conference on Universal Usability (New York, NY, USA, 2000), 65-71.

[39] Mynatt, E.D., Rowan, J., Craighill, S. and Jacobs, A. 2001. Digital Family Portraits: Supporting Peace of Mind for Extended Family Members. Proceedings of the SIGCHI Conference on Human Factors in Computing Systems (New York, NY, USA, 2001), 333-340.

[40] Nasreddine, Z.S., Phillips, N.A., Bédirian, V., Charbonneau, S., Whitehead, V., Collin, I., Cummings, J.L. and Chertkow, H. The Montreal Cognitive Assessment, MoCA: A Brief Screening Tool For Mild Cognitive Impairment. fournal of the American Geriatrics Society. 53, 4, 695-699. DOI:https://doi.org/10.1111/j.1532-5415.2005.53221.x. 
[41] National Research Council of the National Academies 2011. Health Care Comes Home: The Human Factors. National Academies Press.

[42] Nishio, S., Ishiguro, H. and Hagita, N. 2007. Can a Teleoperated Android Represent Personal Presence? A Case Study with Children. Psychologia. 50, (2007), 330-342.

[43] Odetti, L., Anerdi, G., Barbieri, M.P., Mazzei, D., Rizza, E., Dario, P., Rodriguez, G. and Micera, S. 2007. Preliminary experiments on the acceptability of animaloid companion robots by older people with early dementia. 2007 29th Annual International Conference of the IEEE Engineering in Medicine and Biology Society (Aug. 2007), 1816-1819.

[44] Orlov, L.M. Technology for Aging in Place: 2018 Market Overview: 2018. https://www.ageinplacetech.com/files/aip/Market Overview 2018 Final 03-14-2018.pdf\#. Accessed: 2018-04-16.

[45] Parker, A.G. 2013. Designing for Health Activism. interactions. 20, 2 (Mar. 2013), 22-25. DOI:https://doi.org/10.1145/2427076.2427082.

[46] Procter, R., Greenhalgh, T., Wherton, J., Sugarhood, P., Rouncefield, M. and Hinder, S. 2014. The Day-to-Day CoProduction of Ageing in Place. Computer Supported Cooperative Work (CSCW). 23, 3 (Jun. 2014), 245-267. DOI:https://doi.org/10.1007/s10606-014-9202-5.

[47] Procter, R., Wherton, J. and Greenhalgh, T. 2018. Hidden Work and the Challenges of Scalability and Sustainability in Ambulatory Assisted Living. ACM Trans. Comput.-Hum. Interact. 25, 2 (Apr. 2018), 11:1-11:26. DOI:https://doi.org/10.1145/3185591.

[48] Procter, R., Wherton, J., Greenhalgh, T., Sugarhood, P., Rouncefield, M. and Hinder, S. 2016. Telecare Call Centre Work and Ageing in Place. Computer Supported Cooperative Work (CSCW). 25, 1 (Feb. 2016), 79-105. DOI:https://doi.org/10.1007/s10606-015-9242-5.

[49] Riche, Y. and Mackay, W. 2010. PeerCare: Supporting Awareness of Rhythms and Routines for Better Aging in Place. Computer Supported Cooperative Work (CSCW). 19, 1 (Feb. 2010), 73-104. DOI:https://doi.org/10.1007/s10606-0099105-z.

[50] Roberts, C. and Mort, M. 2009. Reshaping what counts as care: Older people, work and new technologies. ALTER European Journal of Disability Research / Revue Européenne de Recherche sur le Handicap. 3, 2 (Apr. 2009), 138-158. DOI:https://doi.org/10.1016/j.alter.2009.01.004.

[51] Roberts, C., Mort, M. and Milligan, C. 2012. Calling for Care: 'Disembodied' Work, Teleoperators and Older People Living at Home. Sociology. 46, 3 (Jun. 2012), 490-506. DOI:https://doi.org/10.1177/0038038511422551.

[52] Sherbourne, C.D. and Stewart, A.L. 1991. The MOS social support survey. Social Science \& Medicine. 32, 6 (Jan. 1991), 705-714. DOI:https://doi.org/10.1016/0277-9536(91)90150-B.

[53] Stacey, C.L. Finding dignity in dirty work: the constraints and rewards of low-wage home care labour. Sociology of Health \& Illness. 27, 6, 831-854. DOI:https://doi.org/10.1111/j.1467-9566.2005.00476.x.

[54] Stacey, C.L. 2011. The Caring Self: The Work Experiences of Home Care Aides. Cornell University Press.

[55] Steele, R., Lo, A., Secombe, C. and Wong, Y.K. 2009. Elderly persons' perception and acceptance of using wireless sensor networks to assist healthcare. International fournal of Medical Informatics. 78, 12 (Dec. 2009), 788-801. DOI:https://doi.org/10.1016/j.ijmedinf.2009.08.001.

[56] Vines, J., Lindsay, S., Pritchard, G.W., Lie, M., Greathead, D., Olivier, P. and Brittain, K. 2013. Making Family Care Work: Dependence, Privacy and Remote Home Monitoring Telecare Systems. Proceedings of the 2013 ACM International foint Conference on Pervasive and Ubiquitous Computing (New York, NY, USA, 2013), 607-616.

[57] Vines, J., Pritchard, G., Wright, P., Olivier, P. and Brittain, K. 2015. An Age-Old Problem: Examining the Discourses of Ageing in HCI and Strategies for Future Research. ACM Trans. Comput.-Hum. Interact. 22, 1 (Feb. 2015), 2:1-2:27. DOI:https://doi.org/10.1145/2696867.

[58] Waycott, J., Davis, H., Vetere, F., Morgans, A., Gruner, A., Ozanne, E. and Kulik, L. 2014. Captioned Photographs in Psychosocial Aged Care: Relationship Building and Boundary Work. Proceedings of the SIGCHI Conference on Human Factors in Computing Systems (New York, NY, USA, 2014), 4167-4176.

[59] Waycott, J., Vetere, F., Pedell, S., Morgans, A., Ozanne, E. and Kulik, L. 2016. Not For Me: Older Adults Choosing Not to Participate in a Social Isolation Intervention. Proceedings of the 2016 CHI Conference on Human Factors in Computing Systems (New York, NY, USA, 2016), 745-757.

[60] Welsh, D., Morrissey, K., Foley, S., McNaney, R., Salis, C., McCarthy, J. and Vines, J. 2018. Ticket to Talk: Supporting Conversation Between Young People and People with Dementia Through Digital Media. Proceedings of the 2018 CHI Conference on Human Factors in Computing Systems (2018), 375:1-375:14.

Received April 2018; revised July 2018; accepted September 2018. 